\title{
Self-regulated hydrodynamical process in halo stars: a possible explanation of the lithium plateau
}

\author{
Sylvie Théado and Sylvie Vauclair \\ Laboratoire d'Astrophysique, 14 av. Ed. Belin, 31400 Toulouse, France
}

\begin{abstract}
It has been known for a long time (Mestel 1953) that the meridional circulation velocity in stars, in the presence of $\mu$-gradients, is the sum of two terms, one due to the classical thermal imbalance $(\Omega$ currents) and the other one due to the induced horizontal $\mu$-gradients ( $\mu$-induced currents, or $\mu$-currents in short). In the most general cases, $\mu$-currents are opposite to $\Omega$-currents. Vauclair (1999) has shown that such processes can, in specific cases, lead to a quasi-equilibrium stage in which both the circulation and the helium settling is frozen. Here we present computations of the circulation currents in halo star models, along the whole evolutionary sequences for four stellar masses with a metallicity of $[\mathrm{Fe} / \mathrm{H}]=-2$. We show that such a self-regulated process can account for the constancy of the lithium abundances and the small dispersion in the Spite plateau.
\end{abstract}

¿From spectroscopic observations, the lithium abundance in main sequence Pop II field stars with effective temperatures larger than $5500 \mathrm{~K}$ is remarkably constant, with a very low dispersion if any (Spite \& Spite 1982; Spite et al. 1996; Bonifacio \& Molaro 1997; Molaro 1999), while large lithium abundance dispersions do occur for Pop I stars. We claim that the reason for this behavior may be due to the self-regulating process in slowly rotating stars as described by Vauclair (1999).

In rotating stars, the equipotentials of "effective gravity" (including the centrifugal acceleration) have ellipsoidal shapes while the energy transport still occurs in a spherically symetrical way. Tke resulting thermal imbalance must be compensated by macroscopic motions: the so-called "meridional circulation"(Von Zeipel 1924; Mestel 1953; Maeder \& Zahn 1998).

In the presence of vertical $\mu$-gradients, the circulation velocity is the sum of two terms, one which does not depend on $\mu$ (the so-called " $\Omega$ currents") and one which gathers the $\mu$ dependent terms (the " $\mu$ currents").

In the present paper, we have computed the $\Omega$-currents and the $\mu$-currents along the evolutionary track of a .75 solar mass halo stars. All the parameters included in the computations are the same as for the solar models (Richard 1999). The horizontal $\mu$ gradients are derived using Zahn (1992) theory of anisotropic turbulence (see Vauclair (1999 and 2000) for details). The lithium variations with time are then computed within the same framework.

The $\mu$-currents increase with time below the convective zone because of helium settling (it also increases in the core because of nuclear reactions). An equilibrium situation soon occurs below the convection zone, for which the two 

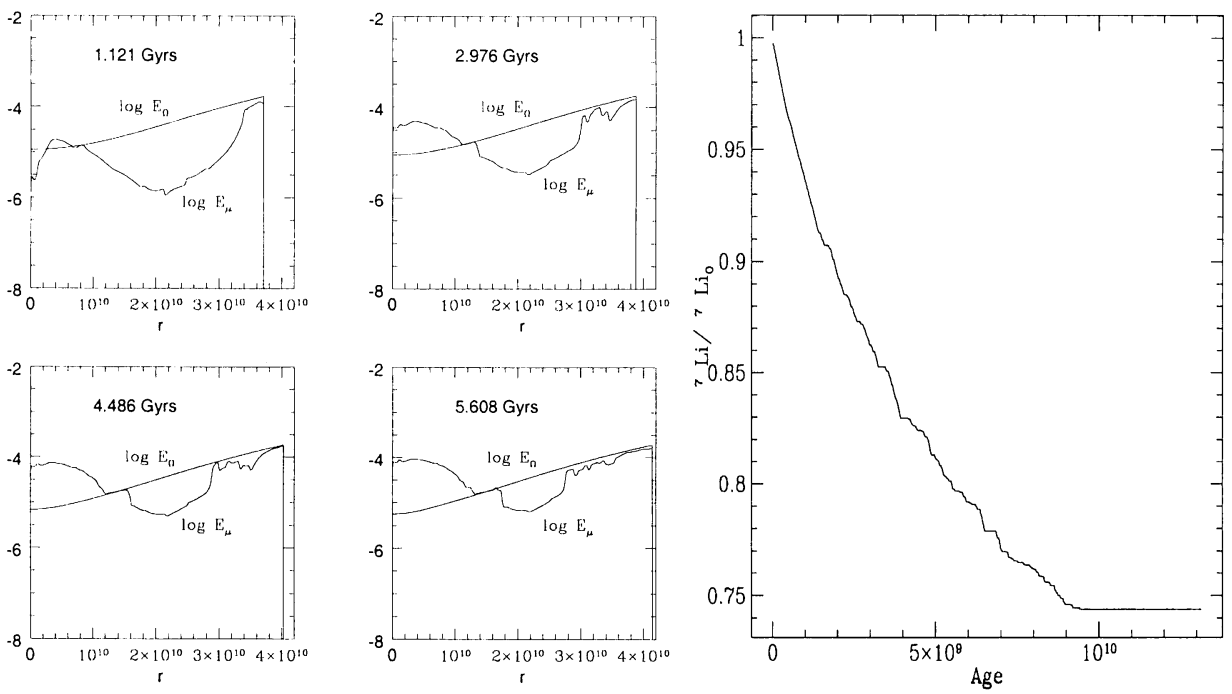

Figure 1. Computations of the $\Omega$-currents and $\mu$-currents and lithium abundance variation with time in a $0.75 M_{\odot}$ halo stars with $[\mathrm{Fe} / \mathrm{H}]=-2$.

currents become equal. Then, the circulation freezes out as well as the gravitational settling. Lithium decreases very slowly and remains constant when the whole star is "frozen". The depletion is not larger than $25 \%$. This can explain the very small dispersion observed in the Spite plateau.

There are many observations in stars which give evidences of mixing processes occuring below the outer convection zones as, for example, the lithium depletion observed in the Sun and in galactic clusters. The process we have described here should not apply in all these stars. The reason could be related to the rapid rotation of young stars on the ZAMS and to their subsequent rotational braking and differential rotation, which is not supposed here to take place in halo stars.

\section{References}

Bonifacio, P., \& Molaro, P. 1997, MNRAS, 285, 847

Maeder, A., \& Zahn, J.-P. 1998, A\&A, 334, 1000

Mestel, L. 1953, Mon. Not. R. Astron. Soc., 113, 716

Molaro, P. 1999, preprint

Richard, O. 1999, phD Thesis, University of Toulouse

Spite, M., \& Spite F. 1982, A\&A, 115, 357

Spite, F., Francois, P., Nissen, P.E., \& Spite, M. 1996, ApJ, 408, 262

Vauclair, S. 1999, A\&A, 351, 973

Vauclair, S. 2000, this conference

Von Zeipel, H. 1924, MNRAS, 84, 665

Zahn, J.-P. 1992, A\&A, 265, 115 


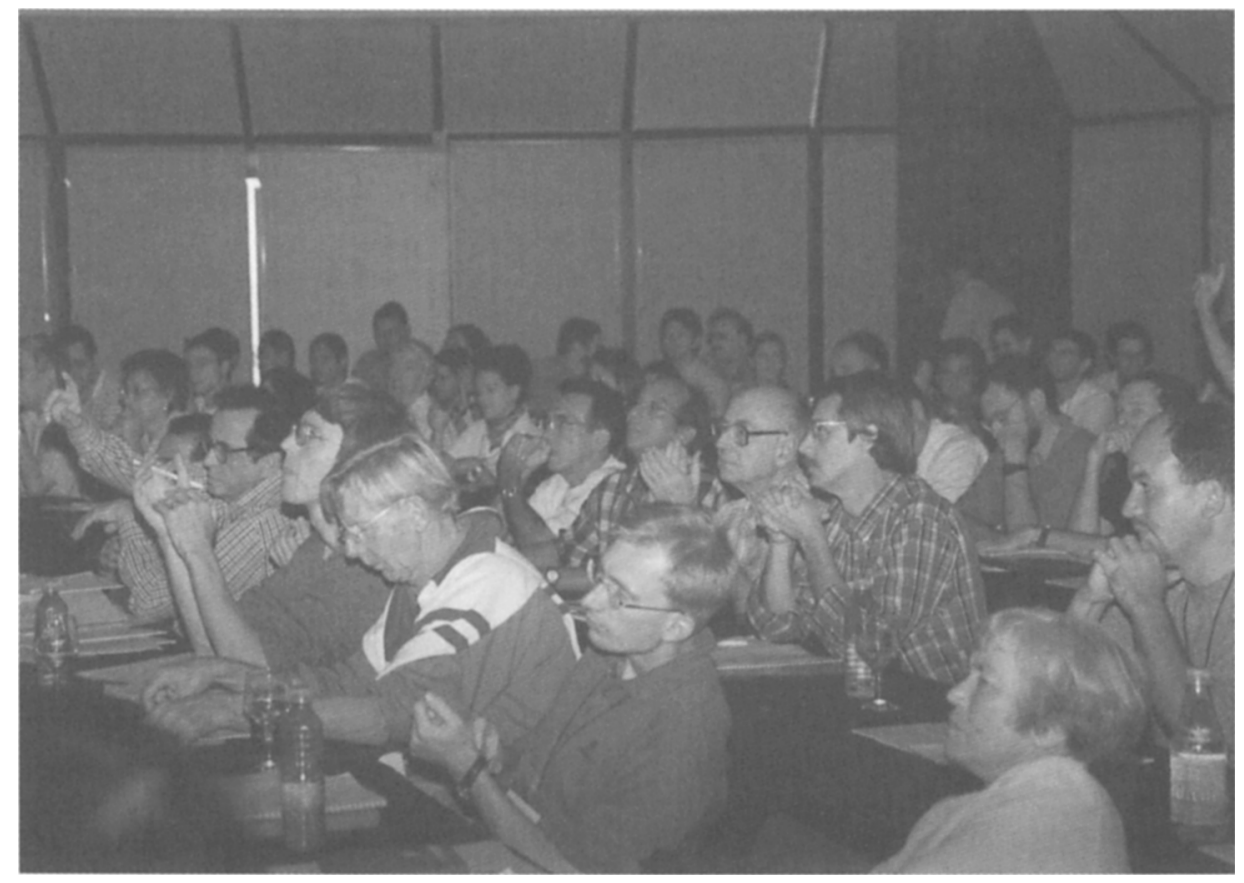

Audience view 